\title{
Knockdown of CXCR7 inhibits proliferation and invasion of osteosarcoma cells through inhibition of the PI3K/Akt and $\beta$-arrestin pathways
}

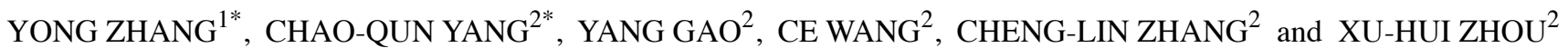 \\ ${ }^{1}$ Department of Orthopedic Surgery, Zhabei District Central Hospital, Shanghai 200070; ${ }^{2}$ Department of Orthopedic Surgery, \\ Changzheng Hospital, Second Military Medical University, Shanghai 200003, P.R. China
}

Received March 31, 2014; Accepted May 23, 2014

DOI: $10.3892 / o r .2014 .3290$

\begin{abstract}
CXC chemokine receptor 7 (CXCR7) has been implicated in tumor development and metastasis in multiple malignancies. Yet, the function and molecular mechanisms of CXCR7 in human osteosarcoma (OS) are still unclear. The aim of the present study was to investigate the role of CXCR7 in human OS. The expression of CXCR7 was assessed by immunohistochemical assay using a tissue microarray procedure in 45 cases of OS tissues. A loss-of-function approach was used to observe the effects of lentiviral vector-mediated CXCR7 siRNA (Lv-siCXCR7) on biological behaviors including proliferative activities and invasive potential, as indicated by MTT and Transwell assays in OS (MG-63 and U-2 OS) cells. The results showed that the expression of CXCR7 protein in OS tissues was significantly increased compared to that in adjacent non-cancerous tissues (68.9 vs. 53.3\%, $\mathrm{P}=0.033$ ), and was correlated with the distant metastasis of the tumors $(\mathrm{P}=0.004)$. Knockdown of CXCR7 suppressed proliferation and invasion of OS cells through decreased expression of PI3K, $\mathrm{AKT}, \beta$-arrestin, proliferating cell nuclear antigen (PCNA), and matrix metalloproteinase-9 (MMP-9). In addition, the tumor volume in U-2 OS subcutaneous tumor models treated with Lv-siCXCR7 was significantly smaller than the tumor volume in the negative control group $(\mathrm{P}<0.01)$. Collectively, our findings indicate that upregulation of CXCR7 expression is correlated with distant metastasis of OS, while knockdown of CXCR7 blocks the development of OS cells through inhibition of the PI3K/AKT and $\beta$-arrestin pathways, suggesting that CXCR7 may serve as a potential therapeutic target for the treatment of cancer.
\end{abstract}

Correspondence to: Professor Xu-Hui Zhou, Department of Orthopedic Surgery, Changzheng Hospital, Second Military Medical University, 415 Fengyang Road, Shanghai 200003, P.R. China

E-mail: zhouxuhui201403@163.com

*Contributed equally

Key words: CXCR7, osteosarcoma, growth, invasion

\section{Introduction}

Osteosarcoma (OS) is the most common primary malignant bone carcinoma in children and adolescents (1). Due to the high propensity for lung metastasis, survival of OS patients has not improved over the past 30 years (2). A novel therapeutic strategy is needed for intervention in regards to the unfavorable prognosis and ineffective treatment for OS patients. Cancer is a genetic disease and tumors develop through a multi-step process. Single or multiple mutations in genes related to growth control and metastasis form the molecular genetic basis of malignant transformation and tumor progression. Therefore, identification of molecular targets that can be exploited in the clinic to treat metastatic disease is desperately needed. Chemokines and chemokine receptors have been described to act as important targets for the treatment of cancer (3).

Chemokines, a family of small cytokines that bind to specific G-protein-coupled seven-span transmembrane receptors, play an important role in many physiological and pathological processes (4). In addition to their initially revealed function in cell trafficking and adhesion, CXC chemokine receptors (CXCRs) have been reported to participate in the development, progression and metastasis in a variety of malignant tumors (5). CXC chemokine receptor 7 (CXCR7), as the receptor for CXCL12 with higher binding affinity, is overexpressed in glioma and regulates tumor proliferation via the ERK and AKT pathways (6,7). Based on the cellular localization, positive expression of CXCR7, mainly detected in the cytoplasm, mediates anti-apoptotic effects in glioma cells and indicates a poor prognosis in gallbladder cancer $(8,9)$ and renal cell carcinoma (10). These data suggest that CXCR7 is a promising therapeutic target.

Furthermore, the association between CXCR7 expression and clinical and pathological features has been analyzed, indicating that CXCR7 is associated with tumor grade and differentiation. CXCR7 exerts a potential function in tumor progression in pancreatic adenocarcinoma (11) and predicts poor disease-free and disease-specific survival in cervical cancer (12). Some studies have shown that several signaling pathways such as CXCR4 and EGFR assist in CXCR7 signaling transduction promoting tumorigenesis, and that CXCR7 coordinates $\beta$-arrestin-2 and K-Ras-dependent pathways involved 
in the development of pancreatic cancer (13). Stromal-derived factor (SDF)-1-mediated CXCR7 signaling has been found to be expressed in bladder and thyroid cancers and to promote cell migration and metastasis $(14,15)$. Thus, targeted inhibition of CXCR7 may provide an effective therapeutic strategy for the treatment of cancer.

However, few studies show that CXCR7 is weakly expressed in malignant hematopoietic cells, and has no enhancing effects on cell proliferation and migration (16). Moreover, it was found that CXCR7 can activate the PI3K/AKT, ERK and STAT3 pathways facilitating the development of bladder cancer (17). To elucidate the function and molecular mechanisms of CXCR7 in human OS, we examined the expression of CXCR7 by immunohistochemical assay using a tissue microarray procedure in OS tissues, and constructed lentiviral vector-mediated Lv-siCXCR7 to observe the effects of CXCR7 on the biological behaviors of OS cells in vitro and in vivo. We hypothesized that CXCR7 may stimulate the growth and invasion of OS cells through regulation of the PI3K/AKT and $\beta$-arrestin pathways.

\section{Materials and methods}

Materials. The OS cell lines (MG-63 and U-2 OS) used for the experiments were obtained from the Institute of Biochemistry and Cell Biology (Shanghai, China). Lentiviral-mediated CXCR7 siRNA (Lv-siCXCR7), negative control vector, and virion-packaging elements were purchased from GeneChem (Shanghai, China). Human OS tissues and the corresponding adjacent non-cancerous tissues (ANCT) were collected from patients at the Department of Orthopedic Surgery, Changzheng Hospital. The tissue microarray of OS was made by Shanghai Outdo Biotech Co. Ltd. (Shanghai, China). All of the antibodies were purchased from Cell Signaling Technology (Boston, MA, USA). CXCR7 primer was synthesized by ABI (Framingham, MA, USA).

Drugs and reagents. Dulbecco's modified Eagle's medium (DMEM) and fetal bovine serum (FBS) were purchased from Thermo Fisher Scientific Inc. (Waltham, MA, USA); TRIzol reagent and Lipofectamine 2000 were obtained from Invitrogen (Carlsbad, CA, USA); M-MLV Reverse Transcriptase was purchased from Promega (Madison, WI, USA); SYBR-Green Master Mix was obtained from Takara (Otsu, Japan); and the ECL Plus kit was obtained from GE Healthcare (Piscataway, NJ, USA).

Clinical samples and data. The tissue microarray was prepared for immunohistochemical analysis (IHC). Human OS tissues and the corresponding ANCT were obtained from biopsies in a total of 45 consecutive OS cases admitted to our hospital from January 2005 to December 2011. The baseline characteristics of the patients before neo-adjuvant chemotherapy are summarized in Table I. The study was approved by the Medical Ethics Committee of the Second Military Medical University and written informed consent was obtained from the patients or their parents before sample collection. Two pathologists respectively reviewed all of the cases.

Tissue microarray. The advanced tissue arrayer (ATA-100; Chemicon International, Temecula, CA, USA) was used to create holes in a recipient paraffin block and to acquire cylindrical core tissue biopsies with a diameter of $1 \mathrm{~mm}$ from the specific areas of the 'donor' block. The tissue core biopsies were transferred to the recipient paraffin block at defined array positions. The tissue microarrays contained tissue samples from 45 formalin-fixed paraffin-embedded cancer specimens with known diagnosis, and the corresponding ANCT from these patients. The block was incubated in an oven at $45^{\circ} \mathrm{C}$ for $20 \mathrm{~min}$ to allow complete embedding of the grafted tissue cylinders in the paraffin of the recipient block, and then stored at $4^{\circ} \mathrm{C}$ until microtome sectioning.

Immunohistochemical staining. Tissue microarray sections were processed for IHC analysis of CXCR7 protein as follows. Immunohistochemical examinations were carried out on 3-mm sections. For anti-CXCR7 immunohistochemistry, unmasking was performed with $10 \mathrm{mM}$ sodium citrate buffer, $\mathrm{pH} 6.0$, at $90^{\circ} \mathrm{C}$ for $30 \mathrm{~min}$. For anti-CXCR7 immunohistochemistry, antigen unmasking was not necessary. Sections were incubated in $0.03 \%$ hydrogen peroxide for $10 \mathrm{~min}$ at room temperature to remove endogenous peroxidase activity, and then in blocking serum [0.04\% bovine serum albumin (A2153; Sigma-Aldrich, Shanghai, China) and 0.5\% normal goat serum (X0907; Dako Corporation, Carpinteria, CA, USA) in phosphate-buffered saline (PBS)] for $30 \mathrm{~min}$ at room temperature. Anti-CXCR7 antibody was used at a dilution of 1:200. The antibody was incubated overnight at $4^{\circ} \mathrm{C}$. Sections were then washed three times for $5 \mathrm{~min}$ in PBS. Non-specific staining was blocked with $0.5 \%$ casein and $5 \%$ normal serum for $30 \mathrm{~min}$ at room temperature. Finally, staining was developed using diaminobenzidine substrate, and the sections were counterstained with hematoxylin. Normal serum or PBS was used to replace the anti-CXCR7 antibody in the negative controls.

Quantification of protein expression. The expression of CXCR7 was semi-quantitatively estimated as the total immunostaining scores, which were calculated as the product of a proportion score and an intensity score. The proportion and intensity of the staining were evaluated independently by two observers. The proportion score reflected the fraction of positive staining cells $(0$, none; $1, \leq 10 \% ; 2,>10$ to $\geq 25 \% ; 3$, $>25$ to $50 \% ; 4,>50 \%$ ), and the intensity score represented the staining intensity ( 0 , no staining; 1 , weak; 2 , intermediate; and 3 , strong). Finally, a total expression score was given ranging from 0 to 12. Based on the analysis in advance, CXCR7 expression was categorized into two groups: low-level CXCR7 expression (score 0-3) and high-level CXCR7 expression (score 4-12). The scoring was independently assessed by two pathologists.

Cell culture and transfection. OS cell lines were cultured in DMEM supplemented with $10 \%$ heat-inactivated FBS, $100 \mathrm{U} /$ $\mathrm{ml}$ of penicillin, and $100 \mu \mathrm{g} / \mathrm{ml}$ of streptomycin. Cells in this medium were placed in a humidified atmosphere containing $5 \% \mathrm{CO}_{2}$ at $37^{\circ} \mathrm{C}$. Cells were subcultured at a $1: 5$ dilution in medium containing $300 \mu \mathrm{g} / \mathrm{ml} \mathrm{G} 418$ (an aminoglycoside antibody; commonly used stable transfection reagent in molecular genetic testing). On the day of transduction, OS cells were replated at $5 \times 10^{4}$ cells/well in 24 -well plates containing serum-free growth medium with polybrene $(5 \mathrm{mg} / \mathrm{ml})$. When 
reaching 50\% confluency, cells were transfected with recombinant experimental virus or control virus at the optimal MOI (multiplicity of infection) of 50 , and cultured at $37^{\circ} \mathrm{C}$ in $5 \%$ $\mathrm{CO}_{2}$ for $4 \mathrm{~h}$. The supernatant was then discarded and serum containing growth medium was added. At 4 days post-transduction, the transduction efficiency was measured by the frequency of green fluorescent protein (GFP)-positive cells. Positive and stable transfectants were selected and expanded for further study. The Lv-siCXCR7 vector-infected clone and the negative control vector-infected cells were designated as the Lv-siCXCR7 and the NC group.

Quantitative real-time PCR. To quantitatively determine the mRNA expression level of CXCR7 in OS cells, real-time PCR was performed. Total RNA was extracted from each clone using TRIzol according to the manufacturer's protocol. Reverse transcription was carried out using M-MLV and cDNA amplification was performed using the SYBR-Green Master Mix kit according to the manufacturer's guidelines. The CXCR7 gene was amplified using a specific oligonucleotide primer, and the $\beta$-actin gene was used as an endogenous control. The PCR primer sequences were as follows: CXCR7, 5'-CACCGCATCTCTTCGACTACTCAGATTCAAGAGAT CTGAGTAGTCGAAGAGATGCTTTTTTG-3' and 5'-GATC CAAAAAAGCATCTCTTCGACTACTCAGATCTCTTGA ATCTGAGTAGTCGAAGAGATGC-3'; $\beta$-actin, 5'-CAACG AATTTGGCTACAGCA-3' and 5'-AGGGGTCTACATGGCA ACTG-3'. Data were analyzed using the comparative $\mathrm{Ct}$ method $\left(2^{-\Delta \Delta \mathrm{Ct}}\right)$. Three separate experiments were performed for each clone.

Western blot assay. OS cell lines were harvested and extracted using lysis buffer (Tris-HCl, SDS, mercaptoethanol and glycerol). Cell extracts were boiled for $5 \mathrm{~min}$ in loading buffer, and then an equal amount of cell extracts was separated on $15 \%$ SDS-PAGE gels. Separated protein bands were transferred onto polyvinylidene fluoride (PVDF) membranes, which were subsequently blocked in $5 \%$ skim milk powder. Primary antibodies against CXCR7, p-PI3K, p-AKT, $\beta$-arrestin, PCNA and metalloproteinase-9 (MMP-9) were diluted according to the manufacturer's instructions and incubated overnight at $4^{\circ} \mathrm{C}$. Subsequently, horseradish peroxidase-linked secondary antibodies were added at a dilution of 1:1,000 and incubated at room temperature for $2 \mathrm{~h}$. The membranes were washed 3 times with PBS, and the immunoreactive bands were visualized using the ECL Plus kit according to the manufacturer's instructions. The relative protein levels in the different cell lines were normalized to the concentration of $\beta$-actin. Three separate experiments were performed for each clone.

Cell proliferation assay. Cell proliferation was analyzed using the MTT assay. Briefly, cells infected with the Lv-siCXCR7 virus were incubated in a 96-well plate at a density of $1 \times 10^{5}$ cells/well with DMEM supplemented with $10 \%$ FBS. Cells were treated with $20 \mu \mathrm{l}$ of MTT dye for $0,24,48$ and $72 \mathrm{~h}$, and subsequently incubated with $150 \mu \mathrm{l}$ of DMSO for $5 \mathrm{~min}$. The color reaction was measured at $570 \mathrm{~nm}$ using an enzyme immunoassay analyzer (Bio-Rad, Hercules, CA, USA). The proliferation activity was calculated for each clone.
Transwell invasion assay. Transwell filters were coated with Matrigel $(3.9 \mu \mathrm{g} / \mu \mathrm{l} ; 60-80 \mu \mathrm{l})$ on the upper surface of a polycarbonate membrane (diameter, $6.5 \mathrm{~mm}$; pore size, $8 \mu \mathrm{m}$ ). After incubation at $37^{\circ} \mathrm{C}$ for $30 \mathrm{~min}$, the Matrigel solidified and served as the extracellular matrix for analysis of tumor cell invasion. Harvested cells $\left(1 \times 10^{5}\right)$ in $100 \mu \mathrm{l}$ of serum-free DMEM were added into the upper compartment of the chamber. A total of $200 \mu \mathrm{l}$ of conditioned medium derived from NIH3T3 cells was used as a source of chemoattractant, which was placed in the bottom compartment of the chamber. After $24 \mathrm{~h}$ of incubation at $37^{\circ} \mathrm{C}$ with $5 \% \mathrm{CO}_{2}$, the medium was removed from the upper chamber. The non-invaded cells on the upper side of the chamber were scraped off with a cotton swab. Cells that had migrated from the Matrigel into the pores of the inserted filter were fixed with $100 \%$ methanol, stained with hematoxylin, then mounted and dried at $80^{\circ} \mathrm{C}$ for $30 \mathrm{~min}$. The number of cells invading through the Matrigel was counted in 3 randomly selected visual fields from the central and peripheral portion of the filter by using an inverted microscope (x200 magnification). Each assay was repeated 3 times.

Subcutaneous tumor model and gene therapy. Six-week-old female immunodeficient mice (BALB/c-nu) were bred at the laboratory animal facility (Institute of Chinese Academy of Sciences, Shanghai), and were housed individually in microisolator ventilated cages with free access to water and food. All experimental procedures were performed according to the regulations and internal biosafety and bioethics guidelines of the Second Military Medical University and the Shanghai Municipal Science and Technology Commission. Three mice were injected subcutaneously with $1 \times 10^{7}$ OS cells (U-2 OS) in $50 \mu \mathrm{l}$ of PBS pre-mixed with an equal volume of Matrigel matrix (Becton-Dickinson). Mice were monitored daily and developed subcutaneous tumors. When the tumor size reached $\sim 5 \mathrm{~mm}$ in length, the tumors were surgically removed, cut into 1-2 $\mathrm{mm}^{3}$ pieces, and re-seeded individually into other mice. When the tumor size reached $\sim 5 \mathrm{~mm}$ in length, the mice were randomly assigned to the $\mathrm{NC}$ group and Lv-siCXCR7 group. In the treatment group, $15 \mu \mathrm{l}$ of $\mathrm{Lv}$-siCXCR7 was injected into the subcutaneous tumors using a multi-site injection format. Injections were repeated every other day after initial treatment. The tumor volume every 3 days was measured with a caliper, using the formula: Volume $=(\text { length } \mathrm{x} \text { width })^{2} / 2$.

Statistical analysis. SPSS 20.0 was used for the statistical analysis. Kruskal-Wallis $\mathrm{H}$ and Chi-square tests were used to analyze the expression levels in all groups. One-way analysis of variance (ANOVA) was used to analyze the differences between groups. The LSD method of multiple comparisons was used when the probability for ANOVA was statistically significant. Statistical significance was set at $\mathrm{P}<0.05$.

\section{Results}

Expression of $C X C R 7$ in $O S$ tissues. The expression of CXCR7 protein was evaluated using IHC staining in OS tissues. The different levels of positive expression of CXCR7 protein were detected in OS (Fig. 1A and B) and ANCT tissues (Fig. 1C and D). Positive CXCR7 immunostaining 
Table I. Correlation of CXCR7 expression with clinicopathological factors of the OS patients.

\begin{tabular}{|c|c|c|c|c|}
\hline \multirow[b]{2}{*}{ Variables } & \multirow[b]{2}{*}{ Cases (n) } & \multicolumn{2}{|c|}{$\begin{array}{c}\text { CXCR7 } \\
\text { expression (n) }\end{array}$} & \multirow[b]{2}{*}{ P-value } \\
\hline & & - & + & \\
\hline Total & 45 & 14 & 31 & \\
\hline Age (years) & & & & 0.850 \\
\hline$<20$ & 28 & 9 & 19 & \\
\hline$\geq 20$ & 17 & 5 & 12 & \\
\hline Gender & & & & 0.557 \\
\hline Male & 26 & 9 & 17 & \\
\hline Female & 19 & 5 & 14 & \\
\hline Histology & & & & 0.456 \\
\hline Osteoblastic & 18 & 4 & 14 & \\
\hline Chondroblastic & 15 & 5 & 10 & \\
\hline Fibroblastic & 7 & 2 & 5 & \\
\hline Others & 5 & 3 & 2 & \\
\hline Ennecking staging & & & & 0.780 \\
\hline I & 13 & 5 & 8 & \\
\hline II & 24 & 7 & 17 & \\
\hline III & 8 & 2 & 6 & \\
\hline Distant metastasis & & & & 0.004 \\
\hline No & 18 & 10 & 8 & \\
\hline Yes & 27 & 4 & 23 & \\
\hline
\end{tabular}

CXCR7, CXC chemokine receptor 7; OS, osteosarcoma.

was mainly localized in the cytoplasm of OS tissue cells. According to the CXCR7 immunoreactive intensity, the positive expression of CXCR7 in OS tissues was significantly increased compared with that in ANCT $(\mathrm{P}=0.033)$ (Table II).

Correlation of CXCR7 expression with clinicopathological parameters. The association of CXCR7 expression with various clinical and pathological factors was analyzed. As shown in Table I, increased expression of CXCR7 was closely correlated with the distant metastasis of OS $(\mathrm{P}=0.004)$. However, no significant association was found between CXCR7 expression and other factors including age, gender, histology and Ennecking staging of the tumor (all $\mathrm{P}>0.05$ ).

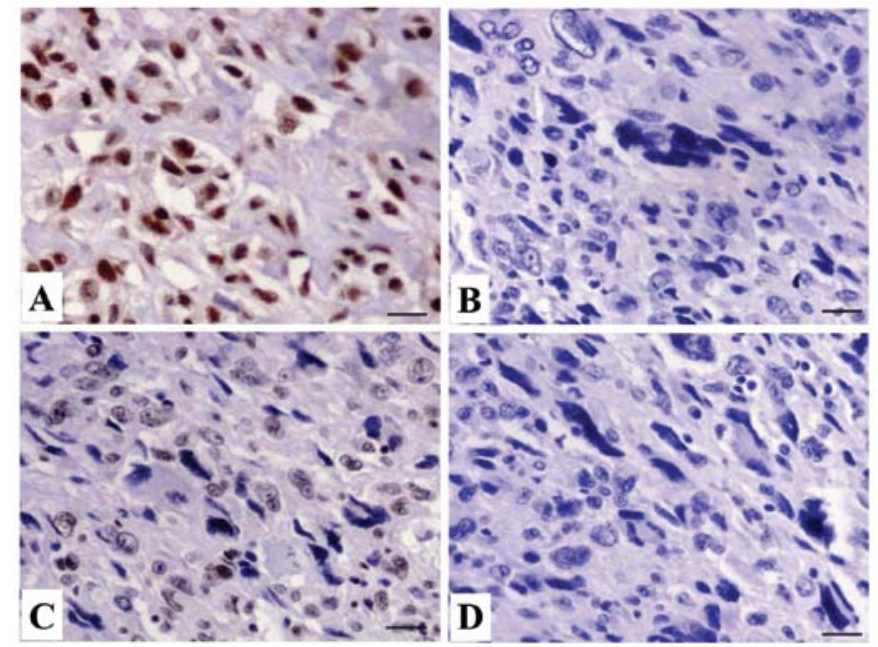

Figure 1. Expression of CXCR7 protein in OS tissues (magnification, $\mathrm{x} 400$ ). OS tissues were immunohistochemically stained with an anti-CXCR7 antibody. Positive expression of CXCR7, mainly localized in the cytoplasm, was increased in OS tissues compared with that in the ANCT. (A) High expression in OS tissues. (B) Low expression in OS tissues. (C) High expression in ANCT tissues. (D) Low expression in ANCT tissues. CXCR7, CXC chemokine receptor 7; OS, osteosarcoma; ANCT, adjacent non-cancerous tissues.

Effect of CXCR7 knockdown on the expression of PI3K, AKT and $\beta$-arrestin. First, lentiviruses of different multiplicity of infections (MOIs) were used to transfect OS cell lines (MG-63 and U-2 OS), and the transfection efficiency of Lv-siCXCR7 (MOI=50) was $>65 \%$. After $\mathrm{Lv}$-siCXCR7 was transfected into the OS cell lines for $24 \mathrm{~h}$, the expression levels of CXCR7 mRNA (Fig. 2A and B) and protein (Fig. 2C and D), p-PI3K, and p-AKT and $\beta$-arrestin proteins (Fig. $2 \mathrm{E}$ and $\mathrm{F}$ ) were detected by real-time PCR and western blot assays, indicating a reduced expression of CXCR7, p-PI3K, p-AKT and $\beta$-arrestin in the Lv-siCXCR7 group when compared with the levels in the $\mathrm{NC}$ group.

Effect of CXCR7 knockdown on cell proliferation. Deregulated cell proliferation is a hallmark of cancer. To verify the effect of CXCR7 on tumor growth in OS cells, we examined cell proliferative activities by MTT assay. The results showed that knockdown of CXCR7 diminished the proliferative activities of OS cells in a time-dependent manner compared to the NC group (Fig. 3A and B). In addition, the expression level of PCNA protein, examined by western blot assay (Fig. 3C and D), was found to be significantly downregulated in the Lv-siCXCR7 group compared with the $\mathrm{NC}$ group.

Table II. Expression of CXCR7 protein in OS tissues.

\begin{tabular}{llcccccccr}
\hline & & \multicolumn{9}{c}{ CXCR7 expression (n) } & & & & \\
\cline { 3 - 6 } Target & Sample & - & + & ++ & +++ & Total & Positive rate (\%) & $\chi^{2}$ & P-value \\
\hline CXCR7 & OS & 14 & 15 & 9 & 7 & 45 & 68.9 & & \\
& ANCT & 21 & 17 & 5 & 2 & 45 & 53.3 & 4.547 & 0.033 \\
\hline
\end{tabular}

OS, osteosarcoma; ANCT, adjacent non-cancer tissue; CXCR7, CXC chemokine receptor 7. 
$\mathbf{A}$
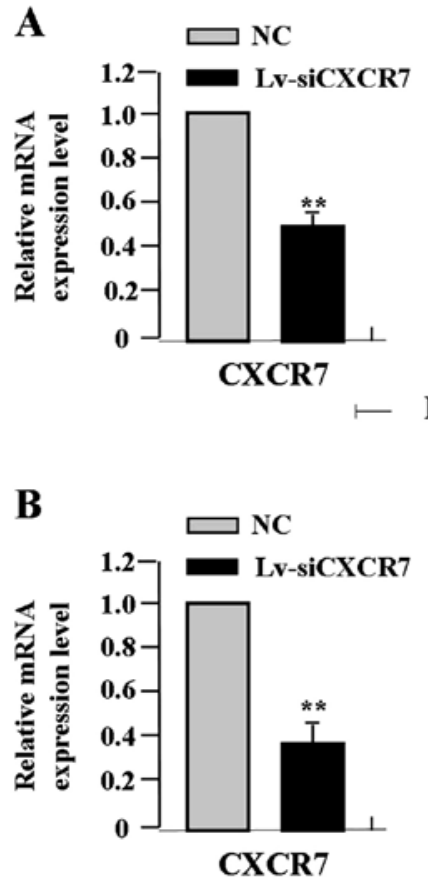

C

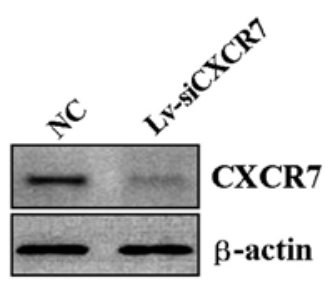

MG-63 -

D

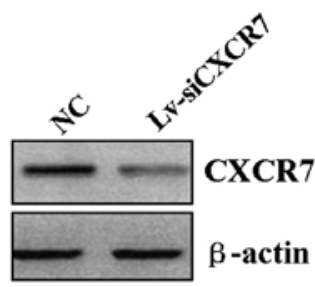

$\mathbf{E}$

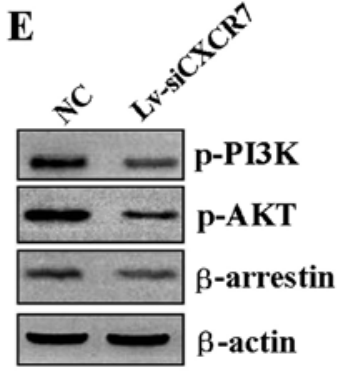

- MG-63 -

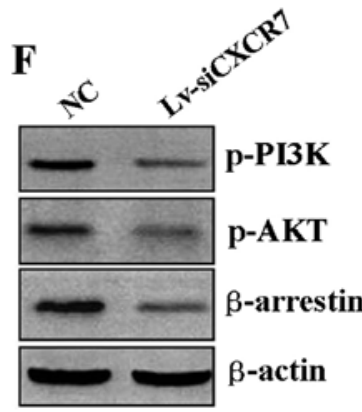

U-2 OS -

Figure 2. Effect of CXCR7 on the expression of PI3K, AKT and $\beta$-arrestin. After OS cells were transfected with Lv-siCXCR7 for $24 \mathrm{~h}$, the expression levels of CXCR7, PI3K, AKT and $\beta$-arrestin were detected by (A and B) real-time PCR and (C-F) western blot assays, revealing decreased expression of CXCR7, PI3K, AKT and $\beta$-arrestin in the Lv-siCXCR7 group when compared with levels in the NC group (each $\left.{ }^{* *} \mathrm{P}<0.01\right)$. CXCR7, CXC chemokine receptor 7; Lv-siCXCR7, lentiviral vector-mediated CXCR7 siRNA; OS, osteosarcoma; NC, negative control.

A

हैं

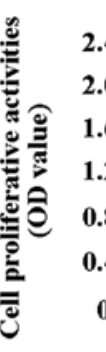

B

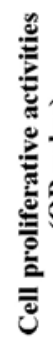

2.4- MG-63

ए $\mathrm{NC}$ LviCXCR7

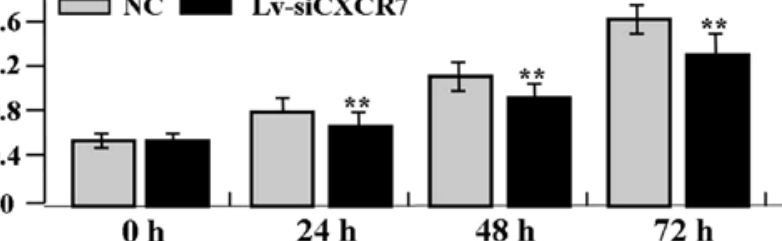

$\mathbf{0 h}$

$24 \mathrm{~h}$

$48 \mathrm{~h}$

$72 \mathrm{~h}$
D

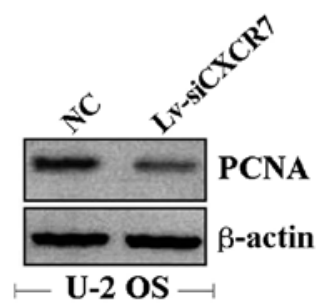

Figure 3. Effect of CXCR7 on cell proliferation. (A and B) MTT assay was used to examine cell proliferative activity revealing that cell proliferation was markedly suppressed in a time-dependent manner in the Lv-siCXCR7 group compared with the NC group (** $\mathrm{P}<0.01)$. (C and D) The expression level of PCNA protein examined by western blot assay was downregulated in the Lv-siCXCR7 group when compared with that in the NC group. CXCR7, CXC chemokine receptor 7; Lv-siCXCR7, lentiviral vector-mediated CXCR7 siRNA; NC, negative control.

Effect of CXCR7 knockdown on cell invasion. To determine the effect of CXCR7 on cell invasion, a Transwell assay was performed. The invasive potential of tumor cells in the
Transwell assay was determined by the ability of cells to invade a matrix barrier containing laminin and type IV collagen, the major components of the basement membrane. 
$\mathbf{A}$

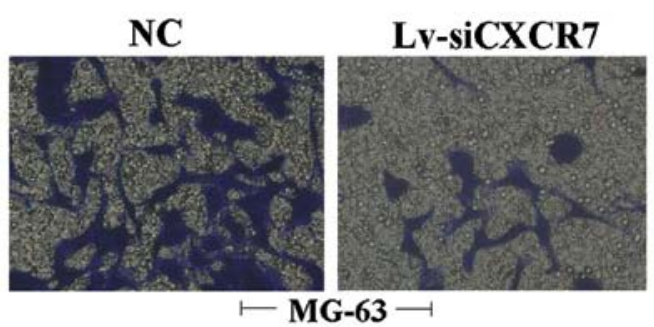

B

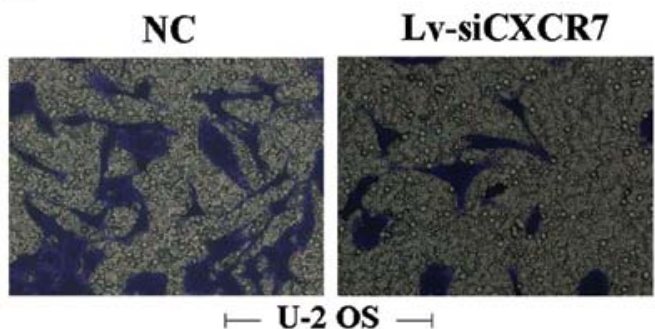

C

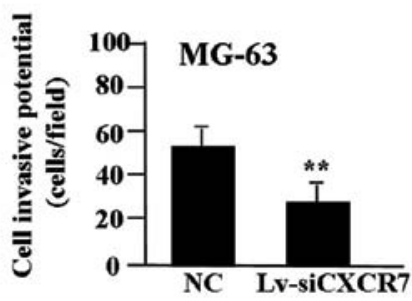

D

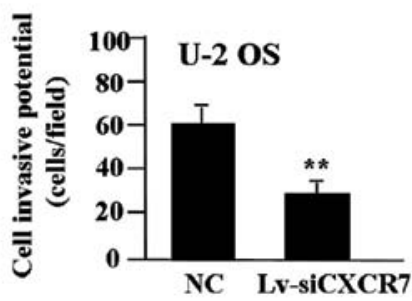

$\mathbf{E}$

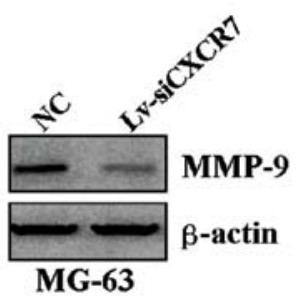

$\mathbf{F}$

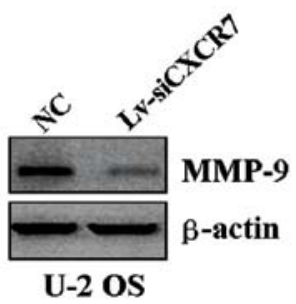

Figure 4. Effect of CXCR7 on cell invasion. (A-D) Transwell assay was performed to determine cell invasion. Cell invasive potential was weakened in the Lv-siCXCR7 group compared with that in the NC group ( $\left.{ }^{* *} \mathrm{P}<0.01\right)$. (E and F) The expression level of MMP-9 protein examined by western blot assay was downregulated in the Lv-siCXCR7 group when compared with the level in the NC group. CXCR7, CXC chemokine receptor 7; Lv-siCXCR7, lentiviral vectormediated CXCR7 siRNA; MMP-9, metalloproteinase-9; NC, negative control.

A
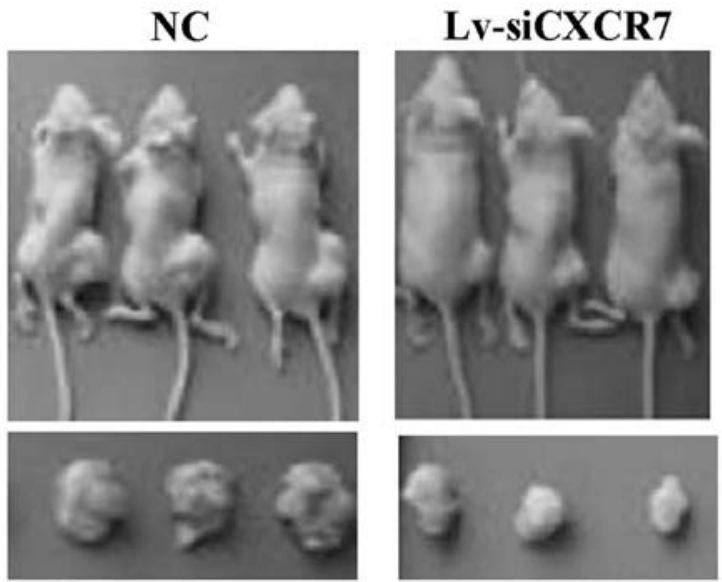

B

C
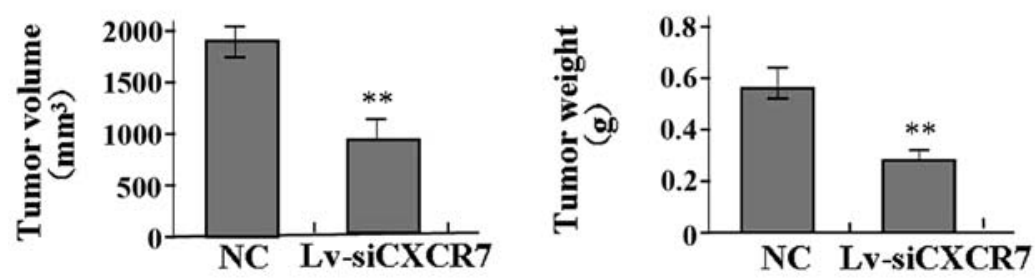

Figure 5. Effect of Lv-siCXCR7 on U-2 OS xenograft tumor growth. (A) The xenograft tumors treated with Lv-siCXCR7 grew substantially slower compared to those in the NC group. The average (B) volume and (C) weight of the tumors in the Lv-siCXCR7 group were significantly reduced than these parameters in the NC group (**P<0.01). Lv-siCXCR7, lentiviral vector-mediated CXCR7 siRNA; OS, osteosarcoma; NC, negative control.

Representative micrographs of the Transwell filters are shown in Fig. 4A and B. The invasive potential of the OS cells was apparently weakened in the Lv-siCXCR7 group compared to the $\mathrm{NC}$ group $(\mathrm{P}<0.01)$ (Fig. 4C and D). In addition, the expression level of MMP-9 protein, examined by western blot assay (Fig. 4E and F), was found to be significantly downregu- lated in the Lv-siCXCR7 group compared with the level in the NC group.

Effect of CXCR7 knockdown on xenograft tumor growth. Our in vitro experiments demonstrated an inhibitory effect of CXCR7 knockdown on OS growth. Therefore, we further 
investigated the effect of CXCR7 on U-2 OS xenograft tumor growth in vivo. The mean volume of the tumors in the experimental mice before treatment was $68.32 \pm 14.25 \mathrm{~mm}^{3}$. During the entire tumor growth period, the tumor growth activity was determined. The tumors treated with $\mathrm{Lv}$-siCXCR7 grew substantially slower compared to the NC group (Fig. 5A). When the tumors were harvested, the average weight and volume of the tumors in Lv-siCXCR7 group were significantly reduced when compared with these parameters in the $\mathrm{NC}$ group (Fig. 5B and C), suggesting that CXCR7 knockdown blocked the growth of OS cells.

\section{Discussion}

CXCR7, a chemokine receptor, has been previously demonstrated to have an impact on growth, apoptosis, invasion/metastasis and prognosis in many types of cancers. CXCR7 was found to be differentially expressed in pancreatic cancer, while knockdown of CXCR7 resulted in decreased migration and invasion (18). The expression of CXCR7 was found to be upregulated in renal cell (10) and human hepatocellular carcinoma (19), and human brain tumors (20). Overexpression of CXCR7 was found to be associated with metastasis and poor survival of patients with hepatocellular carcinoma, indicating its potential function as a therapeutic target (21). CXCR4 and CXCR7 are frequently co-expressed in human cancer tissues and cell lines $(12,13)$. Given the demonstrated importance of CXCR7 in tumor pathogenesis, we further examined the expression of CXCR7 in human OS tissues. The present study showed that CXCR7 was markedly upregulated in the cytoplasm of cells in the OS tissues when compared to the cells in the adjacent non-tumor tissues, and was positively correlated with distant metastasis of OS, suggesting that CXCR7 may represent a new biomarker involved in the development of OS.

Furthermore, the function of CXCR-7 in cancer warrants further research. Some studies have shown that CXCR-7 and IL-8 can contribute to tumor proliferation and metastasis in renal cell carcinoma (22). Activation of CXCR7 on tumor blood vessels facilitates the progression of colon cancer with lung metastasis (23). High expression of CXCR7 also leads to progression, metastasis and angiogenesis in brain tumors, and regulates the transendothelial migration in lymphoma $(24,25)$ To confirm the function of CXCR7 in cancer, our present study showed that knockdown of CXCR7 suppressed the proliferation and invasion of OS cells. Likewise, the findings of Zheng et al support our study, indicating that targeted inhibition of the CXCR7 axis suppresses proliferation, invasion and angiogenesis in hepatocellular carcinoma (26), suggesting that CXCR7 may be a critical therapeutic target for the treatment of cancer.

In response to the regulatory mechanisms of CXCR7 in cancer, a few studies have shown that MAPK, ERK and $\beta$-arrestin-linked signaling pathways are recruited and activated by the CXCR4.CXCR7 complex implicated in cell migration (27). The PI3K/AKT pathway mediates the CXCR7 signaling regulating cell survival and prognosis in head and neck cell carcinoma (28). CXCR7 promotes proliferation and motility in bladder cancer through activation of the AKT and ERK pathways $(17,29)$. Therefore, further study needs to be carried out to investigate the regulatory mechanisms of CXCR7 in OS cells. Our present findings indicated that knockdown of CXCR7 downregulated the expression of p-PI3K, p-AKT and $\beta$-arrestin in OS cells, suggesting that CXCR7 may be involved in the progression of OS via regulation of the PI3K/AKT and $\beta$-arrestin pathways.

PCNA is a nuclear protein that is expressed in proliferating cells and may be required for maintaining cell proliferation, and may be used as a marker for cell proliferation of colon cancer (30). MMP-9 is thought to be a key enzyme involved in the degradation of type IV collagen, and a high level of MMP-9 in tissues is associated with tumor invasion and metastasis (31). It has been reported that the AKT and $\beta$-arrestin pathways promote growth and invasion of tumor cells via regulation of PCNA and MMP-9 expression (32). In the present study, it was found that knockdown of CXCR7 also downregulated the expression of PCNA and MMP-9 in OS cells, suggesting that CXCR7 may promote the progression of OS through AKT and $\beta$-arrestin pathway-mediated regulation of PCNA and MMP-2 expression.

In conclusion, our findings indicate that upregulation of CXCR7 expression is correlated with distant metastasis of OS, and knockdown of CXCR7 inhibits the growth and invasion of OS cells through inhibition of the PI3K/AKT and $\beta$-arrestin pathways, suggesting that CXCR7 may serve as a potential therapeutic target for the treatment of cancer.

\section{References}

1. Chen X, Luther G, Zhang W, et al: The E-F hand calcium-binding protein S100A4 regulates the proliferation, survival and differentiation potential of human osteosarcoma cells. Cell Physiol Biochem 32: 1083-1096, 2013.

2. Roth M, Linkowski M, Tarim J, et al: Ganglioside GD2 as a therapeutic target for antibody-mediated therapy in patients with osteosarcoma. Cancer 120: 548-554, 2014.

3. Goguet-Surmenian E, Richard-Fiardo P, Guillemot E, et al: CXCR7-mediated progression of osteosarcoma in the lungs. $\mathrm{Br}$ J Cancer 109: 1579-1585, 2013.

4. Sun X, Cheng G, Hao M, et al: CXCL12/CXCR4/CXCR7 chemokine axis and cancer progression. Cancer Metastasis Rev 29: 709-722, 2010.

5. Hattermann K and Mentlein R: An infernal trio: the chemokine CXCL12 and its receptors CXCR4 and CXCR7 in tumor biology. Ann Anat 195: 103-110, 2013.

6. Singh AK, Arya RK, Trivedi AK, et al: Chemokine receptor trio: CXCR3, CXCR4 and CXCR7 crosstalk via CXCL11 and CXCL12. Cytokine Growth Factor Rev 24: 41-49, 2013.

7. Odemis V, Lipfert J, Kraft R, et al: The presumed atypical chemokine receptor CXCR7 signals through $\mathrm{G}_{\mathrm{i} / \mathrm{o}}$ proteins in primary rodent astrocytes and human glioma cells. Glia 60: 372-381, 2012.

8. Yao X, Zhou L, Han S, et al: High expression of CXCR4 and CXCR7 predicts poor survival in gallbladder cancer. J Int Med Res 39: 1253-1264, 2011.

9. Hattermann K, Held-Feindt J, Lucius R, et al: The chemokine receptor CXCR7 is highly expressed in human glioma cells and mediates antiapoptotic effects. Cancer Res 70: 3299-3308, 2010.

10. Wang L, Chen W, Gao L, et al: High expression of CXCR4, CXCR7 and SDF-1 predicts poor survival in renal cell carcinoma. World J Surg Oncol 10: 212, 2012.

11. Gebauer F, Tachezy M, Effenberger K, et al: Prognostic impact of CXCR4 and CXCR7 expression in pancreatic adenocarcinoma. J Surg Oncol 104: 140-145, 2011.

12. Schrevel M, Karim R, ter Haar NT, et al: CXCR7 expression is associated with disease-free and disease-specific survival in cervical cancer patients. Br J Cancer 106: 1520-1525, 2012.

13. Heinrich EL, Lee W, Lu J, et al: Chemokine CXCL12 activates dual CXCR4 and CXCR7-mediated signaling pathways in pancreatic cancer cells. J Transl Med 10: 68, 2012. 
14. Gosalbez M, Hupe MC, Lokeshwar SD, et al: Differential expression of stroma derived factor-1 isoforms in bladder cancer. J Urol: Nov 26, 2013 (Epub ahead of print). doi: 10.1016/j. juro.2013.11.053

15. Liu Z, Sun DX, Teng XY, et al: Expression of stromal cell-derived factor 1 and CXCR7 in papillary thyroid carcinoma. Endocr Pathol 23: 247-253, 2012.

16. Tarnowski M, Liu R, Wysoczynski M, et al: CXCR7: a new SDF-1-binding receptor in contrast to normal $\mathrm{CD}^{+} 4^{+}$progenitors is functional and is expressed at higher level in human malignant hematopoietic cells. Eur J Haematol 85: 472-483, 2010.

17. Hao M, Zheng J, Hou K, et al: Role of chemokine receptor CXCR7 in bladder cancer progression. Biochem Pharmacol 84: 204-214, 2012.

18. Guo JC, Li J, Yang YC, et al: Oligonucleotide microarray identifies genes differentially expressed during tumorigenesis of DMBA-induced pancreatic cancer in rats. PLoS One 8: e82910, 2013.

19. Monnier J, Boissan M, L'Helgoualc'h A, et al: CXCR7 is up-regulated in human and murine hepatocellular carcinoma and is specifically expressed by endothelial cells. Eur J Cancer 48: 138-148, 2012.

20. Hattermann K, Mentlein R and Held-Feindt J: CXCL12 mediates apoptosis resistance in rat C6 glioma cells. Oncol Rep 27: 1348-1352, 2012.

21. Xue TC, Han D, Chen RX, et al: High expression of CXCR7 combined with alpha fetoprotein in hepatocellular carcinoma correlates with extra-hepatic metastasis to lung after hepatectomy Asian Pac J Cancer Prev 12: 657-663, 2011.

22. Gahan JC, Gosalbez M, Yates T, et al: Chemokine and chemokine receptor expression in kidney tumors: molecular profiling of histological subtypes and association with metastasis. J Urol 187: 827-833, 2012.
23. Guillemot E, Karimdjee-Soilihi B, Pradelli E, et al: CXCR7 receptors facilitate the progression of colon carcinoma within lung not within liver. Br J Cancer 107: 1944-1949, 2012.

24. Tang T, Xia QJ, Chen JB, et al: Expression of the CXCL12/SDF-1 chemokine receptor CXCR7 in human brain tumours. Asian Pac J Cancer Prev 13: 5281-5286, 2012.

25. Zabel BA, Lewén S, Berahovich RD, et al: The novel chemokine receptor CXCR7 regulates trans-endothelial migration of cancer cells. Mol Cancer 10: 73, 2011.

26. Zheng K, Li HY, Su XL, et al: Chemokine receptor CXCR7 regulates the invasion, angiogenesis and tumor growth of human hepatocellular carcinoma cells. J Exp Clin Cancer Res 29: 31, 2010.

27. Décaillot FM, Kazmi MA, Lin Y, et al: CXCR7/CXCR4 heterodimer constitutively recruits $\beta$-arrestin to enhance cell migration. J Biol Chem 286: 32188-32197, 2011.

28. Liu FY, Zhao ZJ, Li P, et al: NF- $\kappa B$ participates in chemokine receptor 7-mediated cell survival in metastatic squamous cell carcinoma of the head and neck. Oncol Rep 25: 383-391, 2011.

29. Yates TJ, Knapp J, Gosalbez M, et al: C-X-C chemokine receptor 7: a functionally associated molecular marker for bladder cancer. Cancer 119: 61-71, 2013.

30. Risio M: Cell proliferation in colorectal tumor progression: an immunohistochemical approach to intermediate biomarkers. J Cell Biochem (Suppl) 16G: 79-87, 1992.

31. Hornebeck W, Lambert E, Petitfrère E and Bernard P: Beneficial and detrimental influences of tissue inhibitor of metalloproteinase-1 (TIMP-1) in tumor progression. Biochimie 87: 377-383, 2005.

32. Dong S, Kong J, Kong F, et al: Insufficient radiofrequency ablation promotes epithelial-mesenchymal transition of hepatocellular carcinoma cells through Akt and ERK signaling pathways. J Transl Med 11: 273, 2013. 